\title{
Pole coordinates and shape of 30 asteroids
}

\author{
C. Blanco and D. Riccioli \\ Institute of Astronomy, University of Catania, Viale A. Doria 6, 95125 Catania, Italy
}

Received February 14, 1996; accepted March 10, 1998

\begin{abstract}
To obtain a statistically reliable sample of minor planets with known rotation axis orientation and axes ratios, a selection of photometric lightcurves sufficiently covered to give the elements needed for applying the computation methods of the rotational elements was made.

Using the data reported in the "Amplitude-longitude $(A-\lambda)$ plot catalogue of asteroids" (Riccioli \& Blanco $1995)$ as a starting point, the amplitude-magnitude $(A M)$ method (Zappalà et al. 1983a) was adopted.

Due to the poor data available, it was possible to apply the $(A M)$ method only to 30 asteroids. For more than half of these objects no previous determination of pole coordinates and shape exists in the literature.
\end{abstract}

Key words: asteroids

\section{Introduction}

The availability of a statistically reliable sample of asteroids with well-determined rotation axis orientation and axes ratios is essential for the statistical and theoretical modelling of their collisional evolution. The knowledge of the rotation axis distribution becomes more interesting in the case of the asteroid families, because it is possible to ascertain how the fragments of the parent body are distributed in space upon fragmentation. For these reasons, in the last years some parts of observational asteroid researches have been focused on the collection of photometric lightcurves with the aim of acquiring enough and qualified data to compute the pole coordinates and shape of the asteroids.

A long-term and intense photoelectric observational campaign is in progress at the Astronomy Institute of Catania University, in collaboration with Torino Astronomical Observatory, having as its main aim the integration of the observational data of the asteroids with few or incomplete lightcurves.

Send offprint requests to: C. Blanco
By means of a careful search in the literature for asteroid lightcurves and of those recorded in our observational campaign, an amplitude-longitude plot catalogue of asteroids was compiled (Riccioli \& Blanco 1995). Using the data reported in this catalogue as a starting point, we utilized the amplitude-magnitude $(A M)$ method (Zappalà et al. 1983a) based on the assumption of triaxial ellipsoid shape of the asteroid, rotating around the shorter axis. From the lightcurves, we obtain the magnitude $V$ at the maximum of the lightcurve and the amplitude $A$, depending on the rotation axis orientation and on the shape of the asteroid, respectively. The ratio between the two greater axes of the approximating ellipsoid can be obtained from the plot $(A-\lambda)$, if we have a continuous and good distribution in longitude of the observed amplitudes.

Due to the scarce availability of lightcurves from which the amplitude at suitable longitudes can be obtained, it was possible to apply the $(A M)$ method only to 30 asteroids. Of these objects we report the observed and theoretical amplitude-longitude plots and the found values of the pole coordinates and of the axes ratios. For more than half the objects, this is the first determination of the rotation axis orientation and shape.

\section{Photoelectric observations and reduction}

To increase the observational data contained in the literature, mainly in the "Asteroid Photometric Catalogue" (Lagerkivst et al. 1987a) and its Updates (Lagerkvist et al. 1989, 1992), a long-term and intense photoelectric observational campaign was undertaken in collaboration with M. Di Martino and G. De Sanctis of Torino Astronomical Observatory. Besides the purpose of building lightcurves of the asteroids with observational constraints (Di Martino et al. 1994), its aim is to observe asteroids with few or incomplete lightcurves, preferably at suitable ecliptic longitudes to obtain $(A-\lambda)$ plots with well distributed points in longitude.

The photoelectric observations, still in progress, have been carried out, since March 1992, with the 91-cm Cassegrain telescope at M.G. Fracastoro station 
of Catania Astrophysical Observatory (since 28 October 1995 this has been the new name of Serra La Nave stellar station of Catania Astrophysical Observatory). In Table 1 of the "Amplitude-longitude $(A-\lambda)$ plot catalogue of asteroids" (Riccioli \& Blanco 1995), the date of the observational runs and the name of the observed asteroids are reported.

During the 23 runs of observations already made, each lasting on average ten nights, we have used the same instrumentation (photomultiplier, diaphragm, filters, etc...) and observing strategy (Di Martino et al. 1994). The transformation to the standard system was made by means of groups of standard stars, taken from Blanco et al. (1968) and Landolt (1973), usually observed every night on their passage at the meridian. Further information on the reduction and plotting procedure are reported in Di Martino et al. (1994).

\section{Pole and shape determination}

In order to determine the orientation of the rotational axis and the shape of the asteroid, we used the amplitudemagnitude $(A M)$ method suggested by Zappalà (1981) and refined by Zappalà et al. (1983a), which is based on the assumed ellipsoidal shape of the asteroid (with semiaxes $a>b>c$ ) and on the relationships between the aspect angle, the lightcurve amplitude and the asteroid magnitude at the lightcurve maximum, all obtained in several oppositions (at least three). It is important to note that usually the real shape of asteroids is different from the ellipsoidal one and moreover the albedo is often not homogeneous over the entire surface. These discrepancies often lead to conflicting results especially when the data are few.

From the lightcurves, we obtain the magnitude $V$ at the maximum of lightcurve and the amplitude $A$, depending on the rotation axis orientation and on the ratio of the maximum to minimum cross-sections of the asteroid, respectively.

If we assume the smaller axis $c$ to be the asteroid rotation axis, the ratio between the two other axes, and subsequently their single values, can be obtained from the $(A-\lambda)$ plot, if we have a continuous and good distribution in longitude of the observed amplitudes. The modelling curves were obtained using the least square method.

In some cases the extrema of the theoretical curves seem to be overestimated with respect to the observed values. This fact depends on the computing program that, in the absence of observed values at the longitudes of the maximum or the minimum, takes into account the slope of the ascending or descending branches.

From the axes ratios it is possible to obtain the value of the aspect angle (with an uncertain definition of the north or south pole) and hence the pole longitude.

Following Zappalà et al. (1990) suggestions, we have corrected the lightcurve amplitude for its dependence on the phase angle, by means of the relationship $A\left(0^{\circ}\right)=\frac{A(\alpha)}{(1+m \alpha)}$ where $A(\alpha)$ is the observed lightcurve amplitude, $\alpha$ is the solar phase angle and $m$ is a coefficient depending on the asteroid taxonomic class. The $V_{0}(1, \alpha)$ of each asteroid was computed adopting the value $\alpha_{m}$, the arithmetic average of all phase angles.

In Table 1, for each asteroid, the references of the lightcurves used for the estimation of the $V$ magnitude and for the construction of the $(A-\lambda)$ plots are reported. The symbol before the author's name is that used in the corresponding $(A-\lambda)$ plots. Only lightcurves at least $90 \%$ covered were utilized. Due to the available lightcurves, their minimum number (at least three) necessary for applying the $(A M)$ method and to their distribution in longitude, it was possible to compute the pole coordinates and the axes ratios only for 30 asteroids. In Fig. 1, using different symbols for different authors as indicated in Table 1, the $(A-\lambda)$ plots of these asteroids are reported. The $\lambda$ adopted values are the mean values computed over the duration of each lightcurve. The filled symbols indicate the observed values of the amplitude $A$, the empty ones the corresponding values at longitudes $\lambda+180^{\circ}$, the continuous and dashed (in the case of two solutions) lines the theoretical curves.

\section{Results}

According to Zappalà \& Knezevic (1984), the $(A M)$ method for spin-vector determination allows us to obtain a preliminary indication of the rotational properties of the asteroid. One cannot derive the sense of rotation, and it is pratically impossible to distinguish between two pairs of opposite pole solutions, unless the choice takes into account one's stand on the smaller error or on the better fit with the theoretical plot. Notwithstanding this, we preferred to adopt the simple and fast $(A M)$ method, particularly suitable in the case of a very large number of rotation axis and shape determinations.

Table 2 lists the average solar phase angle, the adopted maximum amplitude and the obtained values of the pole coordinates and of the axes ratios of the asteroids to which it was possible to apply the $(A M)$ method. For many asteroids there are two pairs of solutions and it is usually difficult to reject one pair in favour of the other. When this occurs, the two solutions (the $P_{1}$ solution normally has a smaller error than the $P_{2}$ one) differ by about $180^{\circ}$ in ecliptic longitude. Since the data do not distinguish between prograde and retrograde rotation about the same axis, every tabulated solution has a symmetric one with equal probability, which is not reported in the table. The choice between the prograde or the retrograde reported solution was made according to the solution given by the computation program. For some asteroids we obtain errors of the order 1 or 2 degrees that, compared to the few data from which the solution was obtained, appear reasonably low. 
Table 1. References of the lightcurves used for the estimation of the $V$ magnitudes and for the construction of the $(A-\lambda)$ plots. The symbol before the author's name is the same used in the corresponding $(A-\lambda)$ plots

8 Flora

- Ahmad 1954

- van Houten et al. 1958

- Veverka 1971

- Di Martino et al. 1989

- Harris and Young 1989

$$
10 \text { Hygiea }
$$

- Groenveld and Kuiper 1954

- Vesely and Taylor 1985

- Lagerkvist and Williams 1987

- Michalowski et al 1991

- Blanco and Riccioli this paper

\section{Parthenope}

- van Houten 1962

- Wood and Kuiper 1963

- Blanco et al. 1997 14 Irene

- Groenveld and Kuiper 1954

- Wamsteker and Sather 1974

- Scaltriti et al. 1978

- Chang et al. 1981

- Blanco et al. 1997 19 Fortuna

- Wang Chuan-Jin and Liu Dum-Zhag 1965

- van Houten et al. 1979

- Lupishko et al. 1981, 1989

- Weidenschilling et al. 1987, 1990

- Harris et al. 1992

- Blanco et al. 1996

\section{Themis}

4 Degewij et al. 1979

- van Houten-Groeneveld et al. 1979

- Harris et al. 1989

\section{Atalante}

- Harris and Young 1980

- Schober and Schroll 1982

- Di Martino et al. 1987
42 Isis

- Harris and Young 1979

- Debehogne et al. 1982

- Vesely and Taylor 1985

- Hainaut-Rouelle et al. 1995

48 Doris

- Harris and Young 1980

- Debehogne et al. 1982

- Schober and Schroll 1982

66 Maja

- Di Martino et al. 1990

- Blanco and Riccioli this paper

69 Hesperia

- Poutanen et al. 1985

4 Di Martino et al. 1987

- Dotto et al. 1992

- De Angelis and Mottola 1995

- Hainaut-Rouelle et al. 1995

71 Niobe

- Lustig and Dvorak 1975

- Barucci et al. 1985

- Harris and Young 1989

\section{Frigga}

- Lagerkvist and Rickmann 1982

- Zappalà et al. 1983b

- Harris and Young 1989

- Hainaut-Rouelle et al. 1995

$108 \mathrm{Hecuba}$

- Blanco et al. 1994

115 Thyra

- Chang et al. 1981

- Scaltriti et al. 1981

- Mc Cheyne et al. 1985

- Magnusson and Lagerkvist 1991
A comparison between the values found by us and the ones by other authors, mainly computed by different methods, shows a certain agreement.

\section{Flora}

Even if the literature provides many lightcurves, we utilized one value of $A$ by Ahmad (1954); van Houten et al. (1958); Veverka (1971), and Harris \& Young (1989) and two by Di Martino et al. (1989), the only ones showing sure values of the amplitude and giving the magnitude $V$ at the maximum of the lightcurve, necessary to apply the $(A M)$ method. Among the existing solutions of this puzzling asteroid the values by Gehrels \& Owings (1962), by Zappalà et al. (1983b) and Hollis et al. (1987) give partial solutions. A complete solution is given by Di Martino et al. (1989) and De Angelis (1995). All the solutions are in good agreement with that given by us.

\section{Hygiea}

To compute the pole coordinate and shape, 8 values of the amplitude, well distributed in longitude, were 
Table 1. continued

\section{Hermione}

- Di Martino et al. 1987

- Hutton 1990

- Piironen et al. 1994

\ Blanco et al. 1996

150 Nuwa

- Di Martino 1984

^ Blanco et al. 1996

\section{Athor}

- Debehogne and Zappalà 1980

- Carlsson and Lagerkvist 1983

^ Harris and Young 1989

$$
165 \text { Loreley }
$$

- Schober et al. 1988

- Weidenschilling et al. 1990

- Harris et al. 1992

- Shevchenko et al. 1992

196 Philomela

- Yang et al. 1965

¿ Zappalà et al. 1983b

- Erikson et al. 1991

- Licandro et al. 1994

230 Athamantis

$\Delta$ Harris and Young 1980

- Zeigler and Florence 1985

- Harris and Young 1989

- Harris et al. 1992

\section{Honoria}

Lagerkvist et al. 1987b

^ Harris and Young 1989

- Blanco et al. 1996
287 Nephthys

- Scaltriti and Zappalà 1979

- Weidenschillig et al. 1990

- Blanco et al. 1996

334 Chicago

- Zappalà et al. 1989

- Weidenschilling et al. 1990

372 Palma

- Zappalà et al. 1983b

- Haupt 1985

- Weidenschilling et al. 1990

337 Campania

- Tedesco 1979

- Di Martino et al. 1994

A Blanco et al. 1996

386 Siegena

- Zappalà et al. 1982

- Harris and Young 1989

389 Industria

- Haupt 1980

A Magnusson and Lagerkvist 1991

- Lagerkvist et al. 1992

409 Aspasia

Lagerkvist 1981

- Hanslmeier 1982

- Di Martino and Cacciatori 1984

- Hainaut-Rouelle et al. 1995

624 Hektor

- Dunlap and Gehrels 1969

- Hartmann and Cruikshank 1978

^ Dahlgren et al. 1991

- Hainaut-Rouelle et al. 1995

Bel'skaya et al. (1993) who reported only two values of the longitude with one that differs from the solution found by us.

\section{Fortuna}

The $(A-\lambda)$ plot of this asteroid too, even if with many determinations of the amplitude, presents few values at the maximum. Hansen (1977); Morrison (1977) and Lupishko et al. (1985) classify 19 Fortuna as a prograde asteroid. Our values of the pole coordinates and of the axes ratios substantially agree with the ones computed with different methods by Drummond et al. (1988, 1991); Magnusson
The available six values of the amplitude $A$ present an absence in correspondence with the longitude of the maximum. The only solution found in literature comes from utilized. Our results are in agreement with the previous ones found by Michalowski et al. (1991); Erikson \& solution, having the same longitude, but the $\beta_{0}$ value negative. Magnusson (1996) reports synthesis values, with an latitude of the $P_{1}$ solution.

\section{Irene}



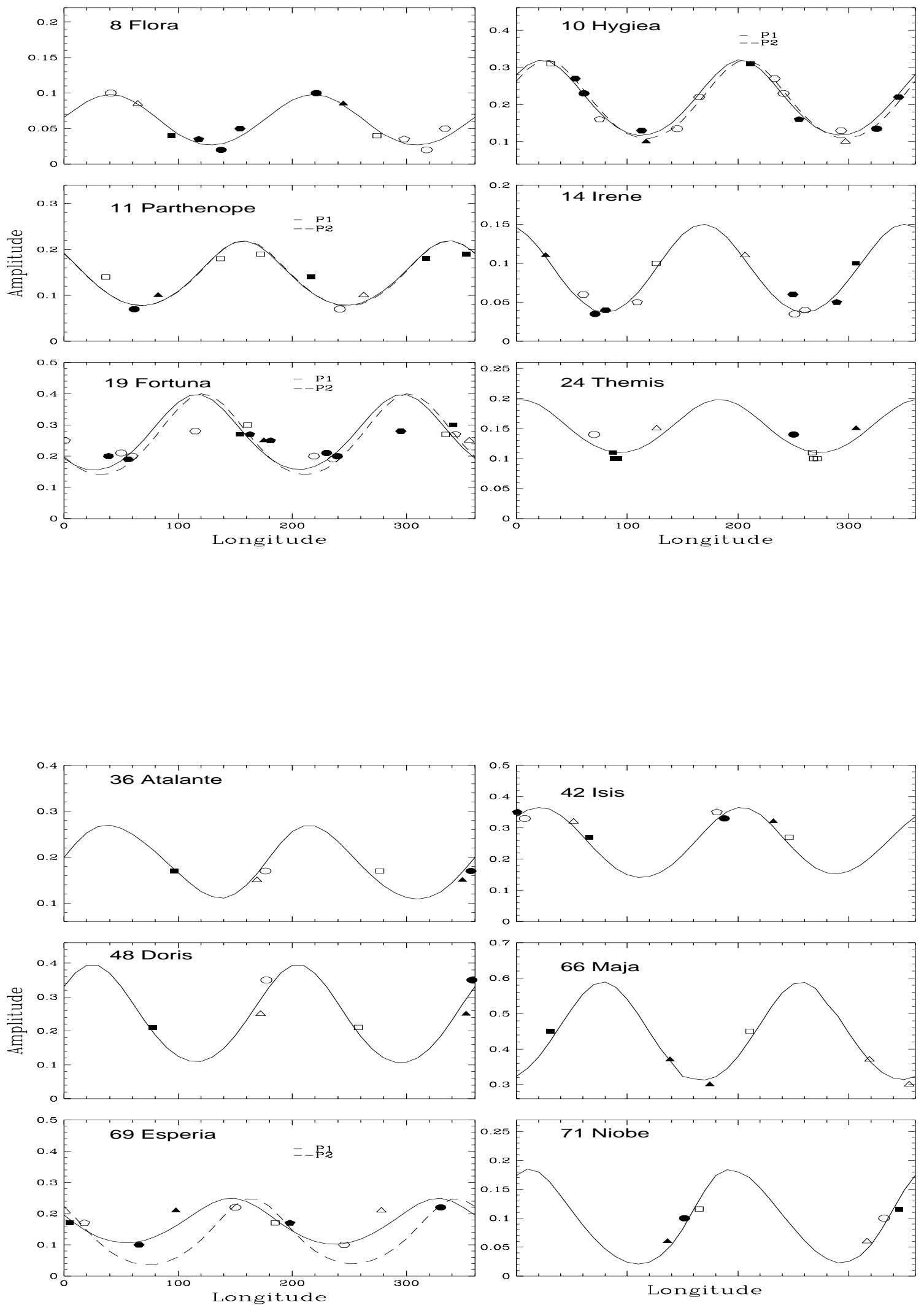

Fig. 1. Amplitude-longitude plots of the asteroid to which it was possible to apply the $(A M)$ method. The filled symbols, as reported in Table 1, indicate the observed values of the amplitude $A$, the empty ones the corresponding ones at longitudes $\lambda+180^{\circ}$, the continuous and (in the case of two solutions) dashed lines the theoretical curves 

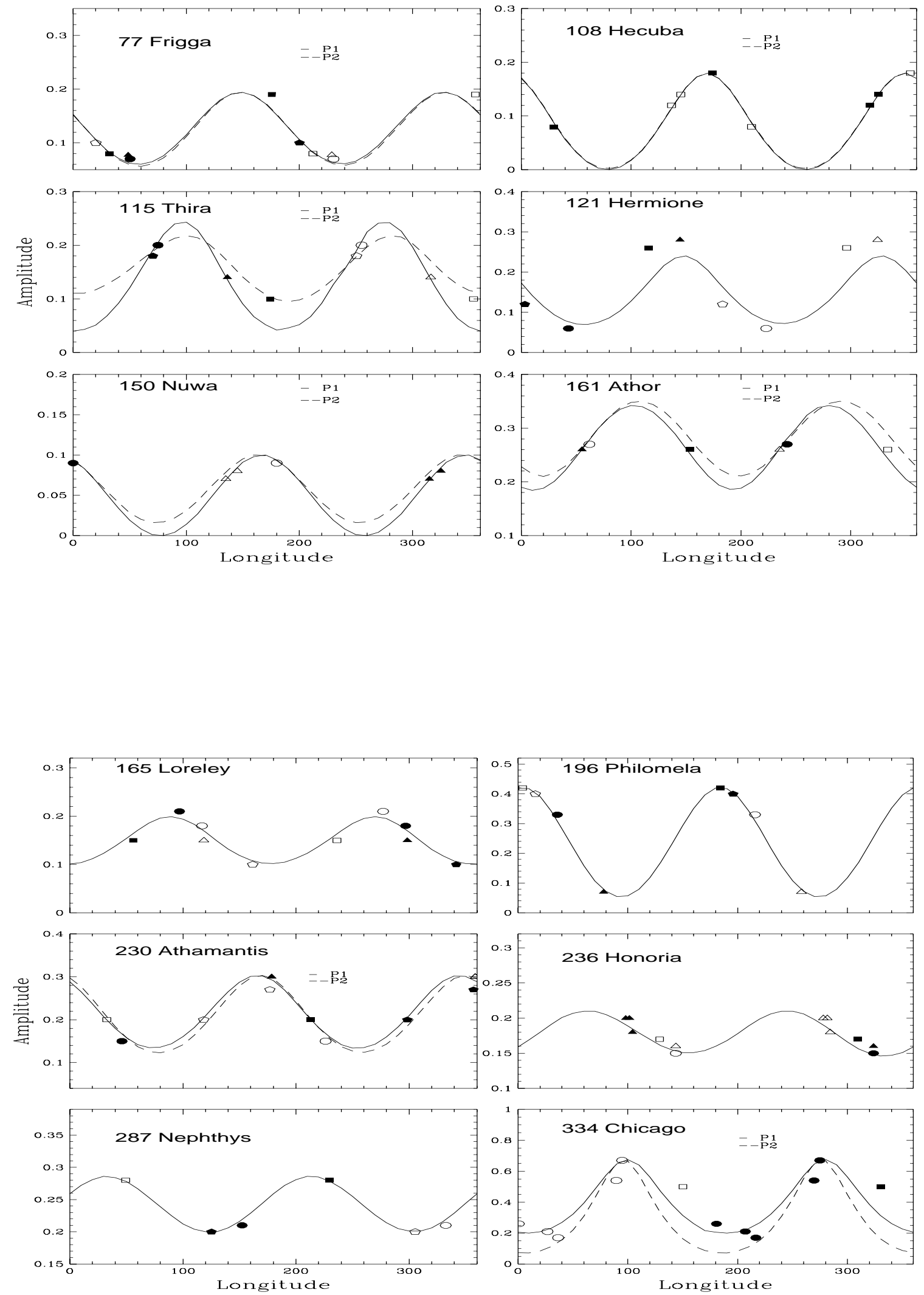

Fig. 1. continued 

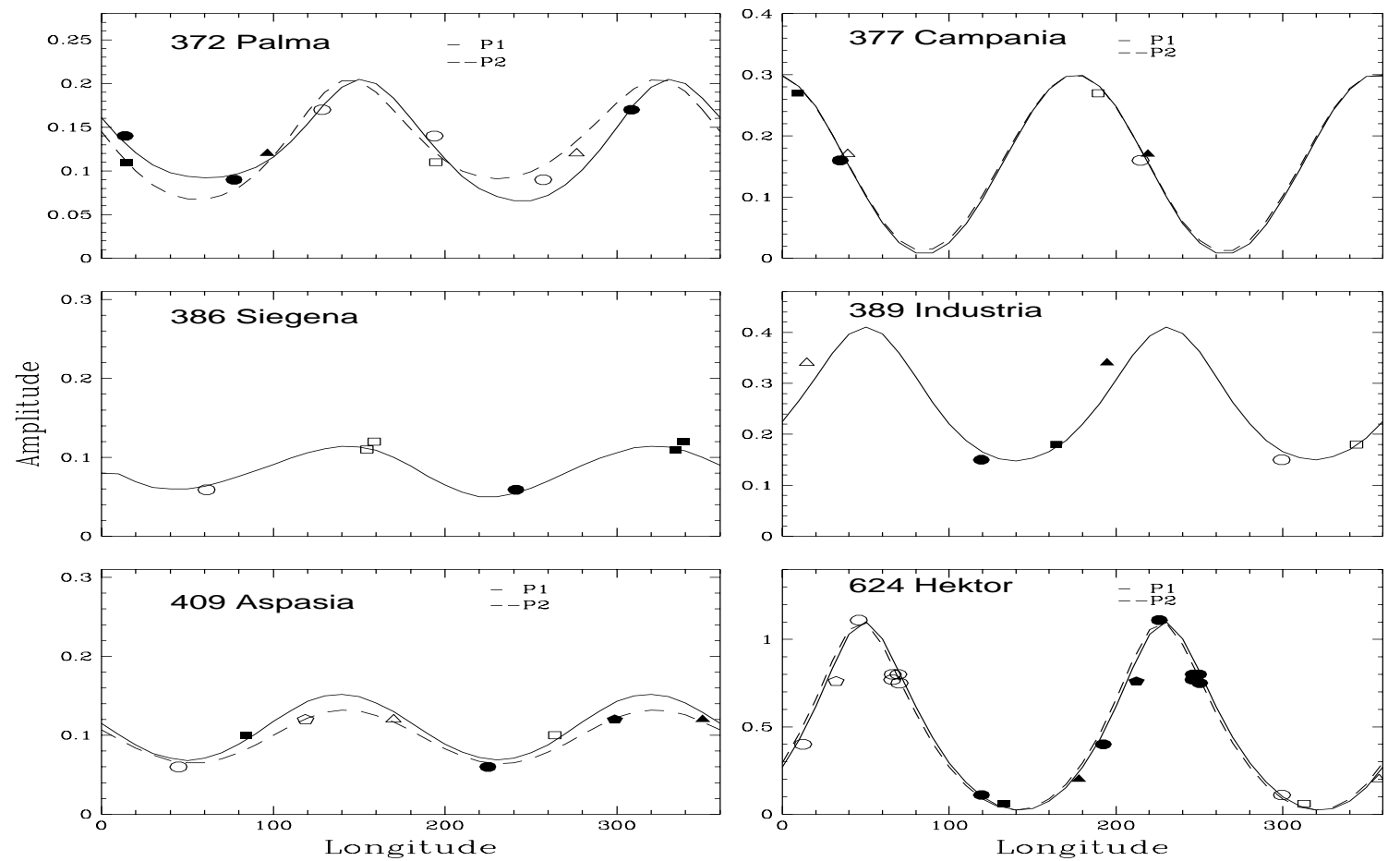

Fig. 1. continued

(1990) and De Angelis (1995). Only our $a / b$ value is a little greater than the other ones. This discrepancy probably results from the overestimated extrema of the theoretical $(A-\lambda)$ plot.

\section{Esperia}

The five values found in literature are well distributed in longitude but show little variations in amplitude. Our pole coordinates differ from those by Velichko et al. (1989) and De Angelis \& Mottola (1995), which are themselves in disagreement. The only existing axes ratio, reported by De Angelis \& Mottola (1995), agrees with those found by us. Krugly \& Velichko (1992) and Magnusson (1996) indicate that 69 Esperia is a prograde rotator.

\section{Thyra}

The four amplitude determinations, available for the $(A-\lambda)$ plot, are distributed in longitude only in an interval of $100^{\circ}$. The only value, among those found by us, that agrees with the existing ones published by Dotto et al. (1995), is a longitude value $\lambda_{0}$.

\section{Hermione}

The available $A$ values of the amplitude are well distributed in longitude. The only value of our solution that agrees with those existing in the literature, reported by De Angelis (1995), is the value of the pole latitude $\beta_{0}$.

\section{Philomela}

The four amplitude determinations utilized to build the $(A-\lambda)$ plot provide well determined extrema and one solution. The existing determinations by Michalowski (1992, 1993); Licandro et al. (1994); De Angelis (1995) and Magnusson (1996), substantially agree with the values of our solution. Except for De Angelis (1995), the other authors obtained two pole solutions that differ by about $180^{\circ}$. The values of the axes ratios are also consistent with ours.

\section{Chicago}

The six lightcurves utilized to obtain the $A$ values are not well distributed in longitude. We obtain two solutions that both differ from the one by Michalowski (1993), the only one found in the literature. The $\lambda_{0}$ values of both solutions found by us are the only ones that agree with the solution by Michalowski (1993).

\section{Industria}

The $(A-\lambda)$ plot was built with only three determinations of the amplitude. The solution found by us is consistent with one of the two solutions published by Michalowski (1993). 
Table 2. Asteroids to which it was possible to apply the $(A-M)$ method. In the columns from left to right, the name of the asteroids, the average solar phase angle, the adopted maximum amplitude, the coordinates of the pole and the axes ratios are reported. When the computation method gives two pairs of solutions, the $P_{1}$ solution normally has a smaller error than the $P_{2}$ one. According to the $(A M)$ method that always gives pairs of opposite solutions, every tabulated solution has a symmetric solution with equal probability

\begin{tabular}{|llccccccc|}
\hline Asteroid & $\alpha_{m}$ & $A_{\max }$ & $\lambda_{0}\left(P_{1}\right)$ & $\beta_{0}\left(P_{1}\right)$ & $\lambda_{0}\left(P_{2}\right)$ & $\beta_{0}\left(P_{2}\right)$ & $a / b$ & $b / c$ \\
\hline 8 Flora & $15^{\circ}$ & 0.10 & $122^{\circ} \pm 3$ & $37^{\circ} \pm 3$ & & & 1.097 & 1.062 \\
10 Hygiea & 7 & 0.32 & $118 \pm 1$ & $44 \pm 1$ & $302 \pm 19$ & $-42 \pm 19$ & 1.343 & 1.144 \\
11 Parthenope & 10 & 0.22 & $73 \pm 7$ & $-51 \pm 5$ & $244 \pm 21$ & $-38 \pm 21$ & 1.225 & 1.208 \\
14 Irene & 10 & 0.15 & $90 \pm 3$ & $-34 \pm 2$ & & & 1.148 & 1.080 \\
19 Fortuna & 10 & 0.30 & $65 \pm 17$ & $49 \pm 9$ & $244 \pm 16$ & $48 \pm 10$ & 1.445 & 1.096 \\
24 Themis & 5 & 0.19 & $274 \pm 33$ & $52 \pm 33$ & & & 1.191 & 1.148 \\
36 Atalante & 10 & 0.27 & $119 \pm 3$ & $-19 \pm 3$ & & & 1.282 & 1.000 \\
42 Isis & 10 & 0.38 & $122 \pm 1$ & $-36 \pm 1$ & & & 1.419 & 1.000 \\
48 Doris & 7 & 0.40 & $113 \pm 6$ & $27 \pm 11$ & & & 1.445 & 1.000 \\
66 Maja & 9 & 0.55 & $162 \pm 3$ & $-50 \pm 1$ & & & 1.660 & 1.000 \\
69 Esperia & 9 & 0.24 & $70 \pm 8$ & $-42 \pm 9$ & $244 \pm 27$ & $-39 \pm 27$ & 1.247 & 1.250 \\
71 Niobe & 5 & 0.20 & $94 \pm 1$ & $-14 \pm 1$ & & & 1.202 & 1.345 \\
77 Frigga & 5 & 0.22 & $57 \pm 4$ & $39 \pm 3$ & $236 \pm 12$ & $-40 \pm 12$ & 1.224 & 1.010 \\
108 Hecuba & 5 & 0.18 & $259 \pm 7$ & $-6 \pm 7$ & $79 \pm 1$ & $13 \pm 11$ & 1.180 & 1.101 \\
115 Thira & 12 & 0.22 & $17 \pm 9$ & $-30 \pm 12$ & $178 \pm 13$ & $-35 \pm 13$ & 1.224 & 1.088 \\
121 Hermione & 7 & 0.28 & $60 \pm 12$ & $-42 \pm 18$ & & & 1.294 & 1.393 \\
150 Nuwa & 10 & 0.10 & $257 \pm 13$ & $1 \pm 13$ & $73 \pm 9$ & $-27 \pm 68$ & 1.097 & 1.015 \\
161 Athor & 19 & 0.34 & $209 \pm 1$ & $47 \pm 1$ & $1 \pm 2$ & $48 \pm 2$ & 1.367 & 0.850 \\
165 Loreley & 5 & 0.19 & $159 \pm 18$ & $-65 \pm 18$ & & & 1.191 & 1.274 \\
196 Philomela & 7 & 0.42 & $98 \pm 1$ & $-20 \pm 6$ & & & 1.472 & 0.914 \\
230 Athamantis & 12 & 0.23 & $60 \pm 8$ & $-51 \pm 6$ & $271 \pm 17$ & $-44 \pm 17$ & 1.318 & 1.195 \\
236 Honoria & 9 & 0.22 & $178 \pm 14$ & $-66 \pm 14$ & & & 1.225 & 1.142 \\
287 Nephthys & 5 & 0.29 & $99 \pm 2$ & $54 \pm 1$ & & & 1.306 & 1.207 \\
334 Chicago & 9 & 0.80 & $0 \pm 14$ & $-59 \pm 9$ & $198 \pm 47$ & $-46 \pm 47$ & 2.089 & 1.742 \\
372 Palma & 13 & 0.20 & $241 \pm 12$ & $7 \pm 12$ & $44 \pm 15$ & $78 \pm 4$ & 1.202 & 1.066 \\
377 Campania & 5 & 0.30 & $266 \pm 7$ & $0 \pm 7$ & $86 \pm 4$ & $3 \pm 47$ & 1.318 & 0.898 \\
386 Siegena & 7 & 0.12 & $236 \pm 19$ & $-14 \pm 19$ & & & 1.116 & 0.776 \\
389 Industria & 9 & 0.36 & $127 \pm 15$ & $-52 \pm 10$ & & & 1.393 & 1.245 \\
409 Aspasia & 10 & 0.14 & $73 \pm 6$ & $48 \pm 4$ & $216 \pm 14$ & $35 \pm 14$ & 1.137 & 1.080 \\
624 Hektor & 7 & 1.11 & $316 \pm 3$ & $+3 \pm 8$ & $147 \pm 13$ & $20 \pm 13$ & 2.779 & 1.000 \\
\hline
\end{tabular}

\section{Hektor}

The $A$ determinations taken from 10 lightcurves are well distributed in longitude. For this well studied asteroid, 11 authors reported pole coordinates and axes ratios values. We obtain two solutions substantially in agreement with those already published and whose mean value was reported by Magnusson (1996).

Of the other 19 objects no previous determination of rotation axis direction and shape has been found in the literature. To the greater part of these minor planets the $(A M)$ method was applied at the minimum conditions of applicability: only with three amplitude determinations but well distributed in longitude. Nevertheless the use of the $(A M)$ method in critical conditions of applicability does not necessarily mean that the results obtained are unreliable. Also to many of the presented asteroids, for which previous determinations exist in the literature, the $(A M)$ method was applied with only three values of the amplitude, obtaining values in accordance with those al- ready known.

The presented results are the first step in our research program. Even if they are in a preliminary form, we wish to publish them to permit their immediate use. The research continues with dedicated observational campaigns and search in the literature for new published data.

Acknowledgements. The authors would like to thank Ms. D. Recupero for editing this note. Special thanks are due to M. Di Martino and A. Cellino for the many and helpful discussions. The work was partially supported by grant ASI-92-RS-78 from the Italian Space Agency.

\section{References}

Ahmad I.I., 1954, AJ 120, 551

Barucci M.A., Fulchignoni M., Burchi R., D'Ambrosio V., 1985, Icarus 61, 152

Bel'skaya I.N., Dovgopol A.N., Erikson A., Lagerkvist C.-I., Oja T., 1993, A\&AS 101, 507 
Blanco C., Di Martino M., Lazzarin M., Cellino A., Riccioli D., 1994, Astron. Soc. Pac. Conf. Ser. 63, 280

Blanco C., Di Martino M., De Sanctis G., Riccioli D., 1996, Planet. Space Sci. 44, No. 2, 93

Blanco C., Di Martino M., De Sanctis G., Ferreri W., Riccioli D., 1997 (in progress)

Blanco V.M., Demers S., Douglass G.G., Fitzgerald M.P., 1968, Publ. U. S. Naval Obs. 21

Carlsson M., Lagerkvist C.-I., 1983, A\&AS 53, 157

Chang Y.C., Wan-Tsing H., Fu-Yuan K., 1981, Acta Astron. Sin. 22,169

Dahlgren M., Fitzsimmons A., Lagerkvist C.-I., Williams I.P., 1991, MNRAS 250, 115

De Angelis G., 1995, Planet. Space Sci. 43, No. 5, 649

De Angelis G., Mottola S., 1995, Planet. Space Sci. 43, No. 8, 1013

Debehogne H., Surdej A., Surdej J., 1978, A\&AS 32, 127

Debehogne H., Lagerkvist C.-I., Zappalà V., 1982, A\&AS 50, 277

Debehogne H., Zappalà V., 1980, A\&AS 42, 85

Degewij J., Tedesco E.F., Zellner B.H., 1979, Icarus 40, 364

Di Martino M., 1984, Icarus 60, 541

Di Martino M., Cacciatori S., 1984, A\&AS 130, 206

Di Martino M., Zappalà V., De Sanctis G., Cacciatori S., 1987, Icarus 69,338

Di Martino M., Zappalà V., Cellino A., Barucci M.A., Harris A.W., Joung J.W., Zeigler K., 1989, A\&A 223, 352

Di Martino M., Ferreri W., Fulchignoni M., De Angelis G., Barucci M.A., Lecacheux J., Burchi R., Di Paolantonio A., 1990, Icarus 87, 372

Di Martino M., Blanco C., Riccioli D., De Sanctis G., 1994, Icarus 107, 269

Dotto E., Barucci M.A., Fulchignoni M., Di Martino M., Rotundi A., Burchi R., Di Paolantonio A., 1992, A\&AS 95, 195

Dotto E., De Angelis G., Di Martino M., Fulchignoni M., De Sanctis G., Burchi R., 1995, Icarus 117, No. 2, 313

Drummond J.D., Weidenschilling J.S., Chapman C.R., Davis D.R., 1988, Icarus 76, 19

Drummond J.D., Weidenschilling J.S., Chapman C.R., Davis D.R., 1991, Icarus 89, 44

Dunlap J.L., Gehrels T., 1969, AJ 74, 796

Erikson A., Cutispoto G., Debehogne H., Hahn G., Lagerkvist C.-I., Magnusson P., 1991, A\&AS 91, 259

Erikson A., Magnusson P., 1993, Icarus 103, 62

Gehrels T., Owings D., 1962, ApJ 135, 906

Groeneveld I., Kuiper G.P., 1954, ApJ 120, 529

Hainaut-Rouelle M.C., Hainaut O.R., Detal A., 1995, A\&AS 112,125

Hansen O., 1977, Icarus 32, 458

Hanslmeier A., 1982, Mitt. Univ. Graz No. 85

Harris A.W., Young J.W., 1979, Icarus 38, 100

Harris A.W., Young J.W., 1980, Icarus 43, 20

Harris A.W., Young J.W., 1983, Icarus 54, 59

Harris A.W., Young J.W., 1989, Icarus 81, 314

Harris A.W., Young J.W., Bowell E., Martin L.J., Millis R.L., Poutanen M., Scaltriti F., Zappalà V., Schober H.J., Debehogne H., Zeigler K.W., 1989, Icarus 77, 171

Harris A.W., Young. J.W., Dockweiler T., Gibson J., Poutanen M., Bowell E., 1992, Icarus 95, 115

Hartmann W.K., Cruinkshank D.P., 1978, Icarus 36, 353
Haupt H., 1980, Mitt. Univ. Graz No. 69

Haupt H., 1985, Mitt. der Univ. Sternwarte Graz No. 107

Hollis A.J., Bembrick C.S., Dumont M., Miles R., 1987, J. Brit. Astron. Assoc. 97, 220

Hutton R.G., 1990, Minor Planet Bull. 17, 41

Krugly Yu.N., Velichko F.P., 1992, Astron. Tsirk. 1553, 35

Lagerkvist C.-I., Barucci M.A., Capria M.T., Fulchignoni M., Guerriero M., Perozzi E., Zappalà, V., 1987a, Asteroid Photometric Catalogue. Consiglio Nazionale delle Ricerche, Rome

Lagerkvist C.-I., Barucci M.A., Capria M.T., Fulchignoni M., Magnusson P., Zappalà V., 1989, Asteroid Photometric Catalogue, First Update. Consiglio Nazionale delle Ricerche, Rome

Lagerkvist C.-I., Barucci M.A., Capria M.T., Dahlgren M., Erikson A., Fulchignoni M., Magnusson P., 1992, Asteroid Photometric Catalogue, Second Update. Uppsala Astronomical Observatory

Lagerkvist C.-I., 1981, A\&AS 44, 345

Lagerkvist C.-I., Rickman H., 1982, The Moon and Planets 27 , 107

Lagerkvist C.-I., Williams I.P., 1987, A\&AS 68, 295

Lagerkvist C.-I., Hahn G., Magnusson P., Rickman H., 1987b, A\&AS 70, 21

Lagerkvist C.-I., Magnusson P., Debehogne H., Hoffman M., Erikson A., De Campos A., Cutispoto G., 1992, A\&AS 95, 461

Landolt A.U., 1973, AJ 78, 959

Licandro J., Gallardo T., Tancredi G., 1994, Rev. Mex. Astron. Astrofis. 28, 91

Lupishko D.F., Tupieva F.A., Velichko F.P., Kiselev N.N., Chernova G.P., 1981, Astron. Vestn., Tome XV, p. 25

Lupishko D.F., Bel'skaya I.N., Velichko F.P., 1985, Vestn. Kharkov Univ. 278, 51

Lupishko D.F., Tupieva F.A., Velichko F.P., Bel'skaya I.N., 1989, Byull. Inst. Astrofiz. 80, 13

Lustig G., Dvorak R., 1975, Acta Phys. Aust. 43, 89

Magnusson P., 1990, Icarus 85, 229

Magnusson P., 1996 (private communication)

Magnusson P., Lagerkvist C.-I., 1991, A\&AS 87, 269

Mc Cheyne R.S., Eaton N., Meadows A.J., 1985, Icarus 61, 443

Michalowski T., Velichko F.P., Lindgren M., Oja T., Lagerkvist C.-I., Magnusson P., 1991, A\&AS 91, 53

Michalowski T., 1992, in Asteroids, Comets, Meteors 1991, Harris A.W., Bowell E. (eds.). Lunar and Planetary Institute, Houston, p. 417

Michalowski T., 1993, Icarus 106, 563

Morrison D., 1977, Icarus 31, 185

Piironen J., Bowell E., Erikson A., Magnusson P., 1994, A\&AS 106,587

Poutanen M., Bowell E., Martin L.J., Thompson D.T., 1985, A\&AS 61,291

Riccioli D., Blanco C., 1995, Catania Astrophys. Obs. Spec. Publ. 143

Scaltriti F., Zappalà V., Stanzel R., 1978, Icarus 34, 93

Scaltriti F., Zappalà V., 1979, Icarus 39, 124

Scaltriti F., Zappalà V., Harris A.W., 1981, Icarus 46, 275

Schober H.J., Scroll A., 1982, A\&A 107, 402

Schober H.J., Di Martino M., Cellino A., 1988, A\&A 197, 327

Shevchenko V.G., Chiornij V.G., Krugly Yu.N., Lupishko D.F., Mohamed R.A., Velichko F.P., Michalowshi T., Avramchuk V.V., Dogvopol A.N., 1992, Icarus 100, 295 
Tedesco E.F., 1979, Ph.D. dissertation, New Mexico State University

van Houten C.J., 1962, Bull. Astron. Inst. Netherlands 16, 160 van Houten-Groeneveld I., van Houten C.J., 1958, ApJ, 127, 253

van Houten-Groeneveld I., van Houten C.J., Zappalà V., 1979, A\&AS 35, 223

Velichko F.P., Lupishko D.F., Shevchenko V.G., 1989, Aston. Tirsk. No. 1541

Veseley C.D., Taylor R.C., 1985, Icarus 64, 37

Veverka J., 1971, Icarus 15, 454

Wamsteker W., Sather R.E., 1974, AJ 79, 1465

Wang Chuan-Jin, Liu Dun-Zhang, 1965, Acta Astron. Sin. 13, 66

Weidenschilling S.J., Chapman C.R., Davis D.R., Greenberg R., Levy D.H., Vail S., 1987, Icarus 70, 191

Weidenschilling S.J., Chapman C.R., Davis D.R., Greenberg R., Levy D.H., Binzel R.P., Vail S.M., Magee M., Spaute
D., 1990, Icarus 86, 402

Wood H.J., Kuiper G.P., 1963, ApJ 137, 1279

Yang Xiu-yi, Zhang You-yi, Li Xiao-qing, 1965, Acta Astron. Sin. 13, 66

Zappalà V., 1981, The Moon and Planets 24, 319

Zappalà V., Scaltriti F., Lagerkvist C.-I., Rickman H., 1982, Icarus 52, 196

Zappalà V., Di Martino M., Farinella P., Paolicchi P., 1983a, Asteroids, Comets, Meteors, Lagerkvist C.I. and Rickman H. (eds.). Uppsala Universitet, Reprocentralen, HSC, Uppsala, pp. 73-76

Zappalà V., Di Martino M., Cellino A., Farinella P., De Sanctis G., Ferreri W., 1989, Icarus 82, 354

Zappalà V., Cellino A., Barucci M.A., Fulchignoni M., Lupishko D.F., 1990, A\&A 231, 548

Zappalà, V., Knezevic Z., 1984, Icarus 59, 436

Zappalà V., Scaltriti F., Di Martino M., 1983b, Icarus 56, 325

Zeigler K.W., Florence W.B., 1985, Icarus 62, 512 